\title{
Synthesis and Evaluation of QS-7-Based Vaccine Adjuvants
}

\author{
Pengfei Wang, ${ }^{* \dagger}$ Đani Škalamera, ${ }^{\dagger}$ Xianwei Sui,${ }^{\dagger}$ Ping Zhang, ${ }^{*+}$ and Suzanne M. Michalek ${ }^{\S}$
}

†Department of Chemistry, ${ }^{\star}$ Department of Pediatric Dentistry, ${ }^{\S}$ Department of Microbiology, University of Alabama at Birmingham, $90114^{\text {th }}$ Street South, Birmingham, AL 35294

Table of Content

\begin{tabular}{|l|l|}
\multicolumn{2}{l}{ Page } \\
\hline Table of content & S1 \\
\hline Serum IgG, IgG1, and IgG2a anti-rHagB data & S2 \\
\hline Serum IgG, IgG1, and IgG2a anti-Dnak data & S2 \\
\hline Weight monitoring of mice & S3-4 \\
\hline${ }^{1}$ H NMR spectra of 5 & S5 \\
\hline${ }^{1}$ H NMR spectra of $\mathbf{6}$ & S6 \\
\hline
\end{tabular}


Table S1. Serum IgG, IgG1, and IgG2a anti-rHagB activity at weeks 2, 4, and 6 (mean, $\mathrm{ng} / \mathrm{mL})^{\mathrm{a}}$

\begin{tabular}{|l|l|l|l|}
\hline IgG & Week 2 & Week 4 & Week 6 \\
\hline rHagB & 13,536 & 179,250 & 994,834 \\
\hline rHagB+GPI-0100 & 176,443 & $1,217,680$ & $1,494,636$ \\
\hline rHagB+QS17-Dac (5) & 164,105 & $1,720,071$ & $1,788,455$ \\
\hline rHagB+QS17-D (6) & 70,014 & 715,327 & $1,129,470$ \\
\hline
\end{tabular}

\begin{tabular}{|l|l|l|l|}
\hline IgG1 & Week 2 & Week 4 & Week 6 \\
\hline rHagB & 11,987 & 237,255 & $1,090,346$ \\
\hline rHagB+GPI-0100 & 154,331 & $1,087,097$ & $1,220,970$ \\
\hline rHagB+QS17-Dac (5) & 155,165 & $1,625,664$ & $1,604,545$ \\
\hline rHagB+QS17-D (6) & 69,126 & 814,128 & $1,219,138$ \\
\hline
\end{tabular}

\begin{tabular}{|l|l|l|l|}
\hline IgG2a & Week 2 & Week 4 & Week 6 \\
\hline rHagB & 194 & 3,150 & 16,268 \\
\hline rHagB+GPI-0100 & 37,410 & 147,219 & 230,959 \\
\hline rHagB+QS17-Dac (5) & 19,558 & 187,366 & 238,286 \\
\hline rHagB+QS17-D (6) & 2,033 & 8,810 & 11,233 \\
\hline
\end{tabular}

${ }^{\mathrm{a}}$ Female BALB/c mice (8-10 weeks of age), $35 \mu \mathrm{g}$ of rHagB, $100 \mu \mathrm{g}$ of adjuvants. Mice were immunized on days 0,14 and 28 . Prior to each immunization and on day 42 post the last immunization, serum was collected

Table S2. Serum $\lg G$, $\lg G 1$, and $\lg G 2 a$ anti-Dnak activity at weeks 2,4 , and $6(\text { mean, } \mathrm{ng} / \mathrm{mL})^{\mathrm{a}}$

\begin{tabular}{|l|r|r|r|}
\hline IgG & week 2 & week 4 & \multicolumn{1}{c|}{ week 6 } \\
\hline DnaK & 107 & 14,427 & 105,278 \\
\hline Dnak+GPI-0100 $(100 \mu \mathrm{g})$ & 1,876 & 566,641 & $1,000,056$ \\
\hline Dnak+QS7-DAc $(100 \mu \mathrm{g})$ & 1,931 & 325,572 & 819,527 \\
\hline Dnak+GPI-0100 $(50 \mu \mathrm{g})$ & 1,276 & 372,068 & 707,551 \\
\hline Dnak+QS7-DAc $(50 \mu \mathrm{g})$ & 545 & 159,762 & 591,740 \\
\hline
\end{tabular}

\begin{tabular}{|l|r|c|c|}
\hline IgG1 & Week 2 & Week 4 & Week 6 \\
\hline DnaK & 134 & 15,625 & 125,416 \\
\hline Dnak+GPI-0100 $(100 \mu \mathrm{g})$ & 1,107 & 636,060 & 992,676 \\
\hline Dnak+QS7-DAc $(100 \mu \mathrm{g})$ & 1,748 & 327,329 & 894,155 \\
\hline Dnak+GPI-0100 $(50 \mu \mathrm{g})$ & 900 & 421,097 & 932,631 \\
\hline Dnak+QS7-DAc $(50 \mu \mathrm{g})$ & 393 & 172,569 & 676,092 \\
\hline
\end{tabular}

\begin{tabular}{|l|c|c|c|}
\hline IgG2a & Week 2 & Week 4 & Week 6 \\
\hline DnaK & 100 & 1,394 & 5,297 \\
\hline Dnak+GPI-0100 $(100 \mu \mathrm{g})$ & 488 & 55,137 & 127,475 \\
\hline Dnak+QS7-DAc $(100 \mu \mathrm{g})$ & 865 & 68,622 & 212,097 \\
\hline Dnak+GPI-0100 $(50 \mu \mathrm{g})$ & 433 & 23,042 & 58,974 \\
\hline Dnak+QS7-DAc $(50 \mu \mathrm{g})$ & 223 & 18,862 & 79,068 \\
\hline
\end{tabular}

${ }^{\mathrm{a}}$ Female BALB/c mice (8-10 weeks of age), $20 \mu \mathrm{g}$ of Dnak. Mice were immunized on days 0, 14 and 28. Prior to each immunization and on day 42 post the last immunization, serum was collected 
Table S3. Weight monitoring of mice immunized by s.c. route with $\mathrm{rHagB}$ alone or with adjuvants over time. ${ }^{\mathrm{a}}$

\begin{tabular}{|c|c|c|c|c|c|}
\hline \multirow[b]{2}{*}{ Group } & \multirow[b]{2}{*}{ mice } & \multicolumn{4}{|c|}{ Weight (g) } \\
\hline & & Week 0 & Week 2 & Week 4 & Week 6 \\
\hline \multirow{7}{*}{ no adjuvant } & $\mathrm{A} 1$ & 19.4 & 20.1 & 21.3 & 21.7 \\
\hline & $\mathrm{A} 2$ & 19.0 & 20.0 & 21.1 & 21.6 \\
\hline & $\mathrm{A} 3$ & 20.4 & 22.7 & 23.2 & 23.8 \\
\hline & A4 & 19.2 & 20.5 & 22.0 & 21.3 \\
\hline & A5 & 19.3 & 21.0 & 22.4 & 21.5 \\
\hline & A6 & 19.2 & 20.5 & 22.1 & 21.2 \\
\hline & mean & 19.4 & 20.8 & 22.0 & 21.9 \\
\hline \multirow{7}{*}{ GPI-0100 } & B1 & 17.0 & 17.8 & 18.6 & 19.7 \\
\hline & B2 & 20.5 & 21.8 & 23.0 & 23.8 \\
\hline & B3 & 19.4 & 21.3 & 21.9 & 23.0 \\
\hline & B4 & 17.8 & 19.0 & 20.3 & 20.3 \\
\hline & B5 & 20.6 & 21.7 & 22.0 & 23.1 \\
\hline & B6 & 19.4 & 20.9 & 21.4 & 21.8 \\
\hline & mean & 19.1 & 20.4 & 21.2 & 22.0 \\
\hline \multirow{7}{*}{5} & $\mathrm{C} 1$ & 17.8 & 17.9 & 19.2 & 20.0 \\
\hline & $\mathrm{C} 2$ & 18.0 & 19.2 & 20.9 & 21.2 \\
\hline & $\mathrm{C} 3$ & 19.0 & 19.4 & 20.6 & 21.7 \\
\hline & $\mathrm{C} 4$ & 21.0 & 23.1 & 22.7 & 23.5 \\
\hline & $\mathrm{C} 5$ & 17.8 & 18.5 & 19.7 & 20.0 \\
\hline & C6 & 18.3 & 19.3 & 20.6 & 21.4 \\
\hline & mean & 18.7 & 19.6 & 20.6 & 21.3 \\
\hline \multirow{7}{*}{6} & D1 & 18.1 & 19.6 & 20.9 & 21.4 \\
\hline & D2 & 19.6 & 20.6 & 21.6 & 21.6 \\
\hline & D3 & 18.3 & 20.6 & 20.5 & 20.8 \\
\hline & D4 & 19.3 & 20.4 & 21.5 & 22.2 \\
\hline & D5 & 19.2 & 20.4 & 21.0 & 21.7 \\
\hline & D6 & 18.5 & 19.4 & 21.7 & 20.9 \\
\hline & mean & 18.8 & 20.2 & 21.2 & 21.4 \\
\hline
\end{tabular}

${ }^{\mathrm{a}}$ Female BALB/c mice (8-10 weeks of age), $35 \mu \mathrm{g}$ of rHagB, $100 \mu \mathrm{g}$ of adjuvants.

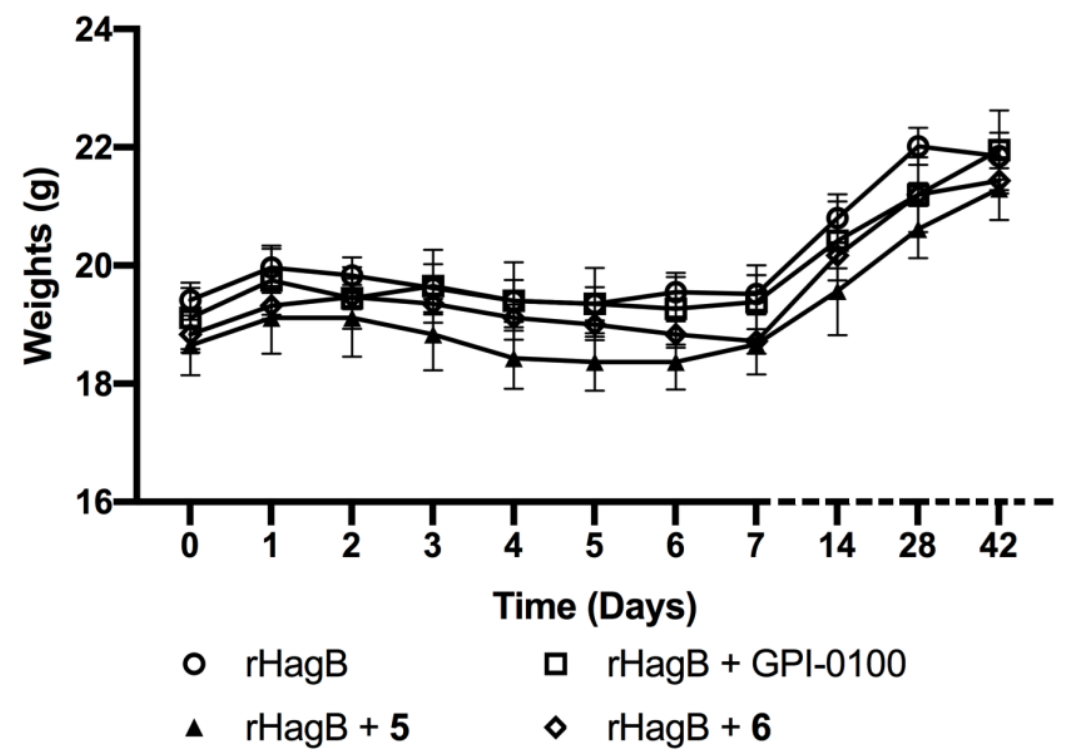

Figure S1. Weight of mice following s.c. immunized with rHagB alone or with adjuvants over time. Mice were immunized on days 0,14 and 28. Mice were weighed prior to each immunization and at 6 weeks following the initial immunization. Values are expressed as mean \pm SEM. 
Table S4. Weight monitoring of mice immunized by s.c. route with Dnak alone or with adjuvants over time. ${ }^{\mathrm{a}}$

\begin{tabular}{|c|c|c|c|c|c|}
\hline \multirow[b]{2}{*}{ Group } & \multirow[b]{2}{*}{ mice } & \multicolumn{4}{|c|}{ Weight (g) } \\
\hline & & Week 0 & Week 2 & Week 4 & Week 6 \\
\hline \multirow{7}{*}{ no adjuvant } & A1 & 19.5 & 19.9 & 20.8 & 22.6 \\
\hline & A2 & 19.1 & 19.4 & 20.4 & 20.8 \\
\hline & A3 & 19.0 & 19.9 & 20.3 & 22.0 \\
\hline & $\mathrm{A} 4$ & 20.1 & 20.4 & 21.6 & 21.9 \\
\hline & A5 & 20.3 & 21.1 & 22.6 & 23.6 \\
\hline & A6 & 20.0 & 20.3 & 22.0 & 22.2 \\
\hline & mean & 19.7 & 20.2 & 21.3 & 22.2 \\
\hline \multirow{7}{*}{$\begin{array}{l}\text { GPI-0100 } \\
(100 \mu \mathrm{g})\end{array}$} & B1 & 18.1 & 18.7 & 20.7 & 21.4 \\
\hline & B2 & 19.7 & 19.1 & 20.5 & 21.6 \\
\hline & B3 & 19.7 & 19.9 & 22.8 & 21.9 \\
\hline & B4 & 20.7 & 21.7 & 22.8 & 24.0 \\
\hline & B5 & 19.2 & 20.2 & 22.0 & 23.7 \\
\hline & B6 & 20.4 & 20.2 & 21.1 & 22.4 \\
\hline & mean & 19.6 & 20.0 & 21.7 & 22.5 \\
\hline \multirow{7}{*}{$\begin{array}{c}\mathbf{5} \\
(100 \mu \mathrm{g})\end{array}$} & $\mathrm{C} 1$ & 17.7 & 17.8 & 19.0 & 20.2 \\
\hline & $\mathrm{C} 2$ & 19.7 & 20.6 & 20.6 & 22.7 \\
\hline & $\mathrm{C} 3$ & 17.3 & 17.7 & 18.0 & 20.2 \\
\hline & $\mathrm{C} 4$ & 21.8 & 22.1 & 22.3 & 24.0 \\
\hline & C5 & 20.9 & 20.9 & 20.9 & 22.3 \\
\hline & C6 & 20.7 & 20.1 & 20.6 & 22.0 \\
\hline & mean & 19.7 & 19.9 & 20.2 & 21.9 \\
\hline \multirow{7}{*}{$\begin{array}{l}\text { GPI-0100 } \\
(50 \mu \mathrm{g})\end{array}$} & D1 & 19.3 & 19.6 & 21.1 & 21.4 \\
\hline & D2 & 20.2 & 20.8 & 22.0 & 23.0 \\
\hline & D3 & 18.6 & 19.4 & 19.7 & 21.0 \\
\hline & D4 & 19.4 & 21.9 & 22.3 & 22.6 \\
\hline & D5 & 20.5 & 20.8 & 22.7 & 22.5 \\
\hline & D6 & 19.0 & 21.4 & 22.4 & 22.0 \\
\hline & mean & 19.5 & 20.7 & 21.7 & 22.1 \\
\hline \multirow{7}{*}{$\begin{array}{c}5 \\
(50 \mu \mathrm{g})\end{array}$} & E1 & 19.3 & 20.9 & 20.9 & 21.8 \\
\hline & E2 & 20.4 & 20.2 & 21.2 & 22.7 \\
\hline & E3 & 20.0 & 20.0 & 20.7 & 22.0 \\
\hline & E4 & 19.8 & 21.2 & 20.1 & 21.8 \\
\hline & E5 & 20.1 & 20.3 & 22.4 & 22.8 \\
\hline & E6 & 18.8 & 18.2 & 19.3 & 20.5 \\
\hline & mean & 19.7 & 20.1 & 20.8 & 21.9 \\
\hline
\end{tabular}

${ }^{\mathrm{a}}$ Female BALB/c mice (8-10 weeks of age), $20 \mu \mathrm{g}$ of Dnak, $100 \mu \mathrm{g}$ or $50 \mu \mathrm{g}$ of adjuvants.

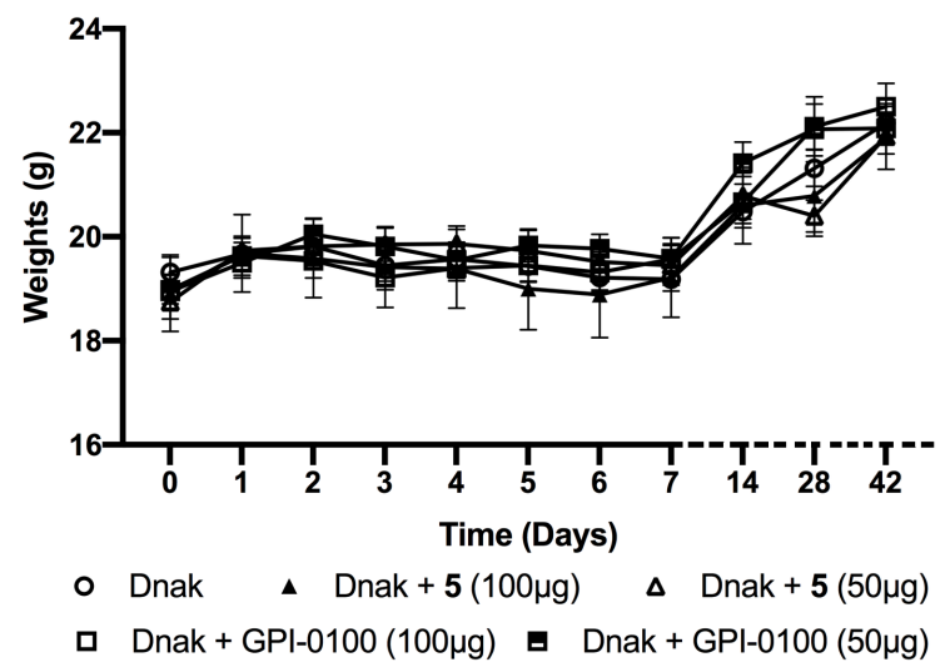

Figure S2. Weight of mice following s.c. immunized with Dnak alone or with adjuvants over time. Mice were immunized on days 0,14 and 28. Mice were weighed prior to each immunization and at 6 weeks following the initial immunization. Values are expressed as mean \pm SEM. 

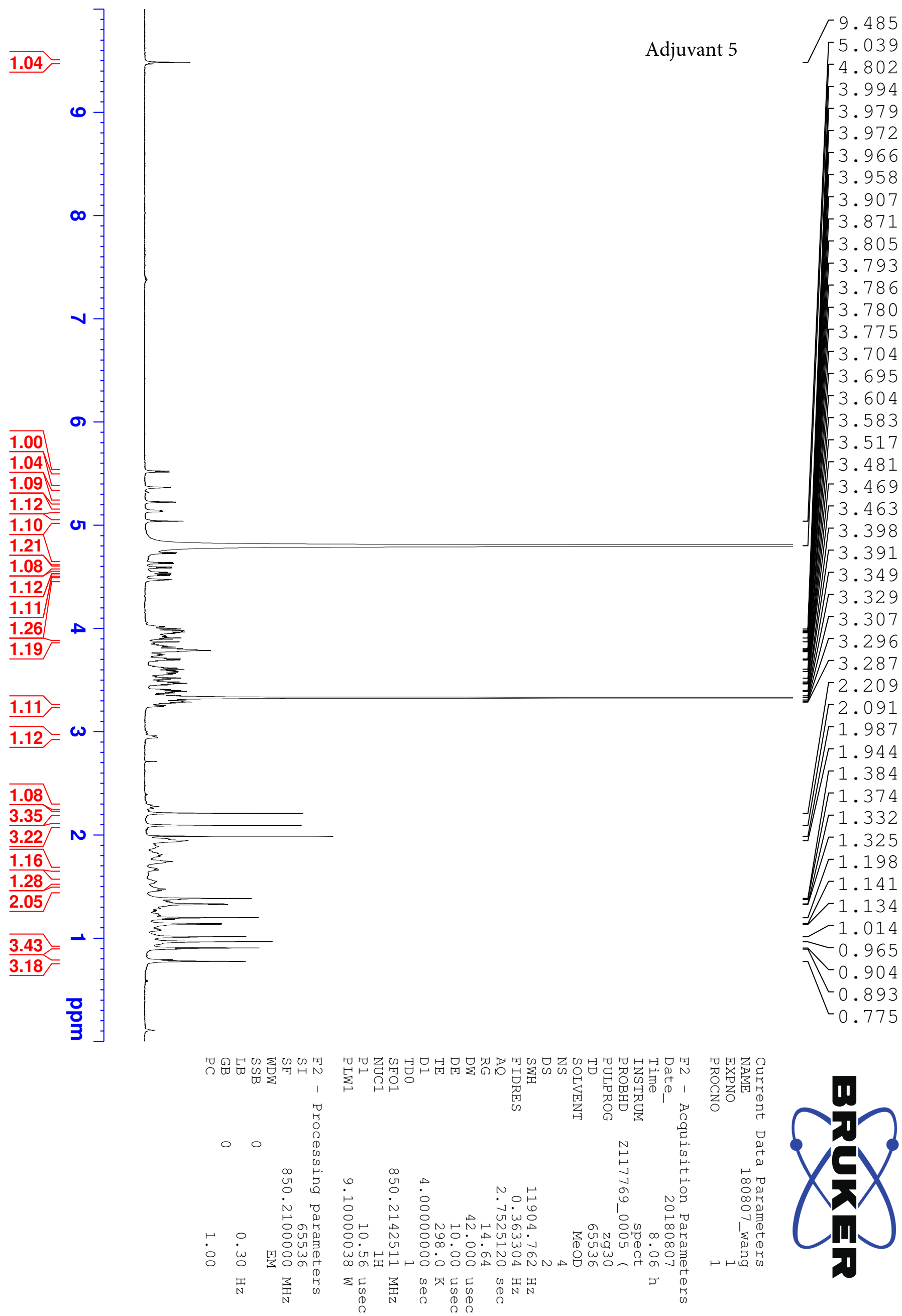


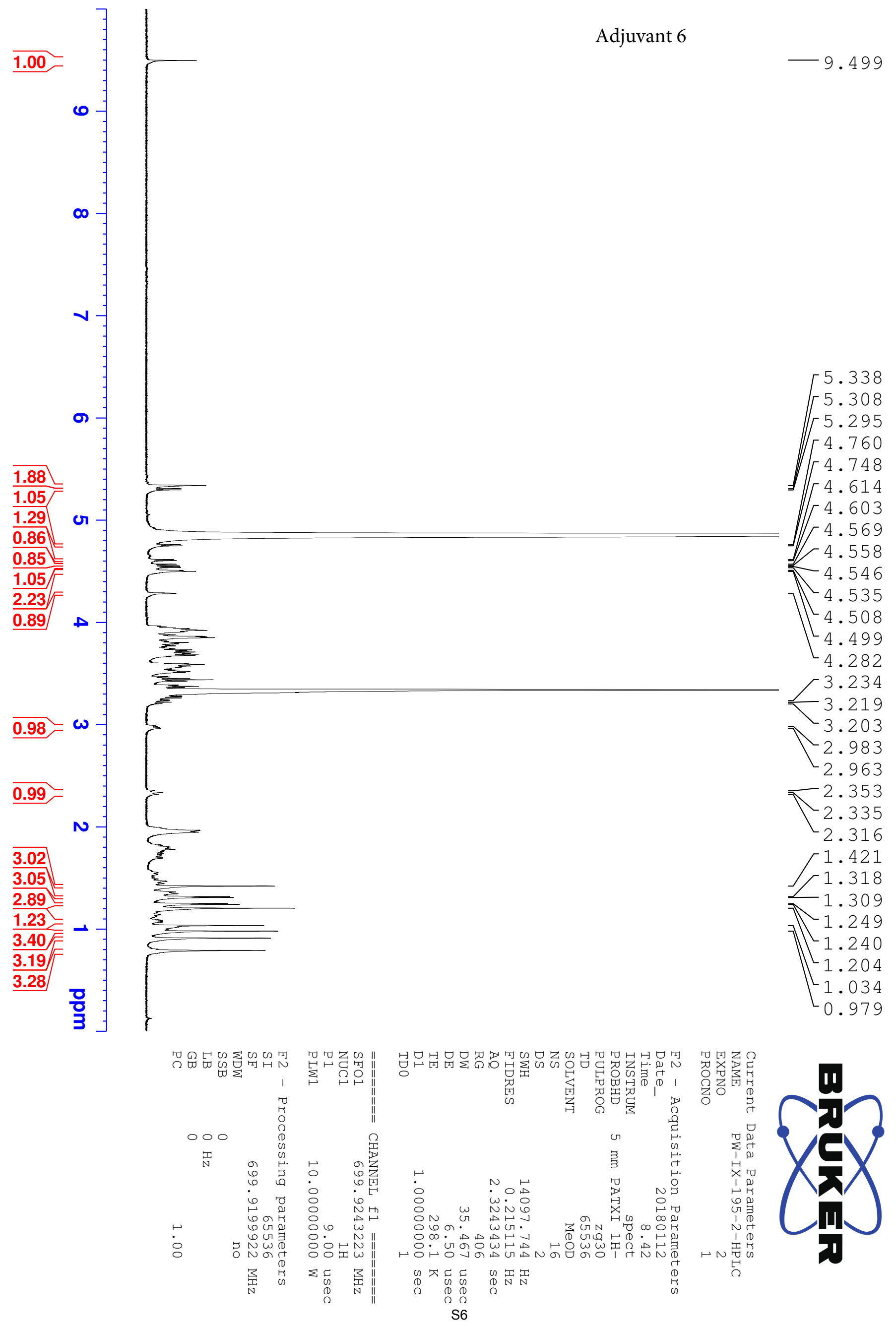

